Magna Scientia Advanced Research and Reviews

(REVIEW ARTIClE)

\title{
Oral care precautions for patients with cystic fibrosis
}

\author{
H. Goumghar* and M. Sidqui \\ Faculty of Dental Medicine of Casablanca, Hassan II University, Casablanca, Morocco.
}

Magna Scientia Advanced Research and Reviews, 2021, 03(02), 073-079

Publication history: Received on 10 November 2021; revised on 13 December 2021; accepted on 15 December 2021

Article DOI: https://doi.org/10.30574/msarr.2021.3.2.0090

\begin{abstract}
Cystic fibrosis (CF) is the most common severe autosomal recessive disease in the Caucasian population. Although it remains incurable, it is currently possible to extend the life expectancy of patients with modern therapeutic possibilities. Given the medical issues that a child with CF faces, oral health may be perceived as being of lesser importance. Thus, the establishment of good dietary and oral hygiene practices may not take place, leading to an increased risk of caries and gingivitis due to poor oral hygiene. A change in patient management may be necessary to ensure optimal care.
\end{abstract}

Keywords: Cystic Fibrosis; Dental Care; Oral health; Preventive dental treatment; Planning dental treatment; Management

\section{Introduction}

Although cystic fibrosis remains incurable, it is currently possible to extend the life expectancy of patients through modern therapeutic possibilities. As cystic fibrosis is no longer the domain of pediatricians, the care of cystic fibrosis patients must be provided by physicians of various specialties. The multidisciplinary team of physicians must include a dentist who is aware of the specific prevention and treatment needs of this patient group.

The maintenance of oral health is important in patients with $\mathrm{CF}$, and it is advisable to educate general dentists and encourage the implementation of a preventive program from birth to reduce the need for treatment, including routine professional care and promotion of the need for very good oral hygiene habits at home [1].

\section{Multidisciplinary management}

The inclusion of a pediatric dentist as part of the multi-professional team managing the care of these children with cystic fibrosis, as they have complex care needs requiring the expertise of specialists, physicians and other health professionals [2]. It is therefore always helpful to discuss treatment plans with the medical team who will have more information about the patient's lung function [3]. In particular, dental practitioners will be asked to examine patients prior to listing them for transplantation to ensure optimal oral health and to reduce the risk of the oral cavity becoming a source of intraoperative sepsis as part of the deep immunosuppression used to prevent graft rejection [4].

\section{Prevention and promotion}

Patients with CF represent a high-priority dental group and should be regularly examined with enhanced prevention[4]. Prevention of dental caries plays a critical role for these patients because of their susceptibility to infection, which influences their quality of life and survival [5]. Prevention is better than cure, both for oral and general health. Therefore, it is important to emphasize key oral health messages from an early age [3], and particularly during the transition from

\footnotetext{
${ }^{*}$ Corresponding author: H Goumghar

Faculty of Dental Medicine of Casablanca, Hassan II University. Casablanca, Morocco.

Copyright $(2021$ Author(s) retain the copyright of this article. This article is published under the terms of the Creative Commons Attribution Liscense 4.0.
} 
childhood to adolescence. It is also advisable to encourage good oral hygiene habits at home[1], schedule regular dental examinations, recommend the use of remineralizing and antibacterial rinses and, if necessary, the use of artificial saliva products [6].

Regular dental examinations are important because preventive care allows dental disease to be managed at an early stage, before restorative treatment is needed. Dentists assess the patient's oral health, monitor growth and development, evaluate the need for any treatment, and make recommendations to reduce the risk of cavities. Visits also include preventive care such as dental cleanings, oral hygiene instruction, dietary counseling, pit and fissure sealants, and topical fluoride applications [7]. Medical care teams should perform regular oral health examinations, learn to recognize the early signs of dental disease, and educate families about the importance of oral health, as well as potential problems that may occur in the oral cavity, and possible treatment and prevention methods [6].

\section{Oral hygiene habits}

Dental biofilm cannot be eliminated, but it can be controlled by regular, daily oral hygiene practices [8]. Patients with cystic fibrosis should follow the general recommendations of health authorities, including the Centers for Disease Control and Prevention, which suggest that patients not share toothbrushes, rinse toothbrushes thoroughly with tap water after brushing, allow the toothbrush to air dry, and do not routinely cover or store the toothbrush in closed containers. Simple oral hygiene recommendations should be discussed, such as more frequent replacement of toothbrushes (once a month) and/or after primary treatment of P. aeruginosa colonization [9]. Children with CF, like all children, should brush twice daily, floss regularly, and visit a dentist annually [10].

\section{Knowledge by dental practitioners}

This disease and its spectrum of clinical manifestations on the affected organs (including the dentition) require increased knowledge by dental practitioners of disease processes, etiology, relevant treatment strategies, and prognosis [11]. Therefore, dental schools should include education on the oral health care needs of patients with CF at the predoctoral level as well as clinical presentations to enable dental students to understand the medical complexities associated with CF treatment [7]. Training should include instruction on potential oral health risks (high-frequency, high-calorie diets), as well as potential positive oral health effects such as long-term antibiotic use [1].

\section{Treatment Planning}

Appropriate dental treatment should be offered to all patients to optimise their general health (Figure 1) [4]. For elective procedures, appointments should be as short as possible, and should be scheduled mid-morning to allow for chest physiotherapy and nebulization in the morning. It is best to schedule the session early in the week to facilitate subsequent management by a specialized cystic fibrosis team [4]. When treating these patients in the dental office, excessive environmental temperatures should be avoided because of the problem of sweating. Patients should be treated in a more upright position to allow for easier drainage of secretions [12] [13].

For children with limited stamina or ability to cooperate, the intraoral examination can be performed at the same time as scaling and polishing of each dental area. Frequent breaks may be preferable for the child with limited endurance.

Scheduling complete scaling over a series of appointments and more frequent preventive or treatment reminders may be a beneficial and appropriate modification of the typical dental protocol. Sonic and ultrasonic scalers should be used with caution in children with cystic fibrosis.

A simple, unflavored prophylactic paste can be used in the presence of ketogenic dietary restrictions, but because of the presence of sweeteners, fluoride varnish is often avoided [14].

\section{Controlling cross-infection}

In patients with $\mathrm{CF}$, the oral microbiota may have an impact on the development of invasive respiratory infections [15]. It is important that they do not develop chronic dental sepsis as part of their care. Even if such a patient is on prophylactic antibiotics, sources of dental infection should be eliminated to keep them as free as possible and not add to the burden of dealing with other areas of actual or potential infection [12]. Segregation of patients with CF is essential in any health care setting to prevent the potential transmission of harmful respiratory pathogens. 
In addition, the dental clinic may present a risk for acquisition of new respiratory organisms. P. aeruginosa was isolated from water samples from dental triple syringes, contra-angles and ultrasonic scalers. The mode of transmission of this organism is unclear, but it establishes that dental equipment may harbor organisms after treatment of patients with $\mathrm{CF}$ and could pose an additional risk to subsequent patients. Another concern is that once dental units are colonized with bacteria, it is difficult to eradicate them completely [4].

\section{Control of oral thrush}

Cystic fibrosis patients attending a routine clinic be screened for risk factors and questioned at 3-month intervals regarding oral thrush symptoms, including frequency of painful or dry mouth, crusty lips, dysphagia, dysphonia or hoarseness, and taste difficulties. Any relationship to antibiotic treatment should be disclosed. Simple screening for risk factors and symptoms would likely lead to improvements in both quality of life and compliance with other cystic fibrosis therapies with the advent of newer and earlier administration of antibacterial therapies [16].

\section{Aesthetics}

Enamel opacities are more common in people with CF. They are thought to be due to a reduced pH resulting in a lack of calcium supply during maturation and thus amelar hypo-mineralization. Tetracycline is known to give children a high incidence of tooth discoloration and hypoplasia, and should therefore be avoided [17]. Most of them need cosmetic improvement as the appearance is quite unacceptable [12].

\section{Medications}

The dental professional should be aware that patients with CF may use bisphosphonates for the treatment of osteoporosis [18]. In addition to the standard history, it is important to take a complete medication history, not only the name of the medication and dosage, but also when and how the medication is taken. Preventive counseling should include information about sugar-containing medications, and efforts should be made to ensure long-term antibiotic therapy and sugar-free vitamin supplements. If they cannot be sugar-free, then appropriate advice on rinsing the mouth with water should be given.

Similarly, if there is evidence of dental erosion, it will be important to know if the child has gastroesophageal reflux disease and is being treated, or is using bronchodilators and needs advice on rinsing with water after their use [3].

Additional risks include portal hypertension-related hypersplenism with resultant thrombocytopenia and the potential for drug-induced marrow suppression, particularly in the setting of acute antibiotic therapy [4].

\section{Coagulopathy}

Patients with CF may have a higher risk of bleeding. Vitamin K deficiency resulting from exocrine pancreatic insufficiency may be subclinical and is common [4]. In a patient with severe pancreatic involvement, it would be wise to check the blood clotting time before surgery [12]. Patients may also have liver involvement, which may affect their clotting abilities [18]. The overall risk of excessive bleeding is low, and risk factors can usually be accurately assessed during history taking [4].

\section{Sedation Treatment}

Any planned treatment under oral, inhaled or intravenous sedation should be performed only after contacting the patient's specialist team to ensure the appropriateness of these techniques and the need for pre- or postoperative optimization of respiratory status, antibiotic treatment or hospitalization, may be required. [4]

The use of sedative agents that interfere with lung function should be avoided, and the patient's physician should be contacted before using nitrous oxide-oxygen sedation in a patient with evidence of severe emphysema [13]. In general, subjects without evidence of respiratory failure or previous hypercapnia (increased carbon dioxide levels) should be suitable for such procedures. Oral premedication with benzodiazepines may result in a risk of hypoventilation with resulting hypoxia and hypercapnia. The risk with nitrous oxide includes the potential for high concentrations of supplemental oxygen to reduce respiratory drive. Prolonged use of sedative agents poses a risk to the ability to clear respiratory secretions, which should be considered [4]. 
Figure 1 Items to elicit from a clinical history when assessing and planning treatment for the person with cystic fibrosis [4]

\section{Checklist for the dental treatment of the patient with cystic fibrosis}

Keep appointments as short as possible and appointment time should be mid-morning to allow for chest physiotherapy and nebuliser morning routines

Treatment planning-if sedation required or indicated liaise with medical team

Be vigilant regarding infection control and list only one person with CF per list

Respiratory

Current clinical status-exacerbation or at baseline?

What is the patient's predominant respiratory organism-note in particular MRSA

Regular bronchodilator use-is pre-treatment indicated?

Current or recent corticosteroid use? Is there a requirement for steroid cover?

Baseline FEV1?

History of respiratory failure?

Current or past supplemental oxygen use?

Current or previous non-invasive ventilation (NIV/BiPap) use?

History of lung transplantation?

If history of current pulmonary exacerbation, low baseline FEV1 (| $40 \%$ predicted), current supplemental oxygen or noninvasive ventilation use or history of lung transplantation - advise liaison with medical team

Bleeding

Is the patient pancreatic insufficient? (Clue-use of supplemental pancreatic enzymes)

Are they prescribed and taking supplemental vitamin K?

History of liver disease?

Use of anticoagulant therapy?

Advise liaison with medical team if significant liver disease/or history of bleeding

Post-procedure analgesia

Is there a history of significant renal disease?

Is there a history of significant liver disease?

Is there a history of broncho-spasm secondary to non-steroidal anti-inflammatories?

Note previous analgesic dosing if available, higher doses may be necessary secondary to hypermetabolism or adjusted for renal/hepatic disease

If considering opioid analgesia-note respiratory history as above. Caution regarding risks of constipation/bowel obstruction

Post-procedure antibiotics

Are post-procedure antibiotics indicated?

Note patient allergy status as there is a higher rate of antibiotic allergy in CF patients

Advise liaison with medical team regarding appropriate antibiotic choice

Considerations for sedation/general anaesthesia

Liaise with medical team prior to any procedure requiring sedation or general anaesthesia

One patient with cystic fibrosis per procedure list

Is pre-operative optimisation of respiratory status advisable-contact medical team to assess requirement for preprocedure antibiotic/physiotherapy management?

Children with cystic fibrosis have dry airways, and the administration of inhalational sedatives can be dangerous when gases are not humidified [12]. In addition, nasal polyps and sinusitis are other common complications of CF that may also make inhalation sedation more difficult [3].

\section{Preanesthetic considerations}

Dental professionals must be aware of the challenges associated with sedation of individuals with cystic fibrosis. They are poor candidates for oral conscious sedation in the office because of compromised lung function. The use of agents that interfere with lung function, such as sedatives and narcotic analgesics, is contraindicated [19]. Close liaison with the patient's medical team is extremely important, not only for the use of medications, but also if surgery or general 
anesthesia (GA) is contemplated, as there is a risk of bleeding tendencies (due to vitamin K malabsorption) or anemia [3].

\subsection{Local anesthesia}

Routine dental treatments pose few problems and can be performed normally without contraindication to local anesthesia [12]. The risk of toxicity from local anesthetics is higher in children because of their smaller size compared to adults. In all children, the maximum recommended dose is $1 / 10$ th canister per kilogram of body weight. However, this dose should be reduced in children with liver disease [3].

The most commonly used local anesthetics in pediatric dentistry are amide agents. Lidocaine hydrochloride (HCl) $2 \%$ with epinephrine $1 / 100,000$ is preferred because of its low allergenic characteristics and greater potency at lower concentrations. Vasoconstrictors are used to constrict blood vessels, counteract the vasodilatory effects of local anesthetic, prolong its duration, reduce systemic absorption and toxicity, and provide a bloodless field for surgical procedures. The use of the vasoconstrictor increases the maximum total dose of the anesthetic agent by nearly $40 \%$. Many agents have been used as vasoconstrictors with local anesthetics. However, none have been shown to be as clinically effective as epinephrine.

Amide-type local anesthetics available for dental use include Lidocaine, Mepivacaine, Articaine, Prilocaine, and Bupivacaine. A long-acting local anesthetic (i.e., bupivacaine) is not recommended for children because of its prolonged effect, which increases the risk of soft tissue injury. It is claimed that Articaine can diffuse through hard and soft tissue from an oral infiltration to provide lingual or palatal soft tissue anesthesia. For minor procedures, local wound infiltration with Bupivacaine reduces the need for opioids, either as an anesthetic adjunct or for postoperative analgesia.

The maximum recommended dose of Lidocaine and Mepivacaine, without vasoconstrictors, for children is $4.4 \mathrm{mg} / \mathrm{kg}$ body weight (Tab.1), and $7 \mathrm{mg} / \mathrm{kg}$ body weight for lidocaine with vasoconstrictors. Thus, for a child weighing $25 \mathrm{~kg}$, the maximum allowable dose of local anesthetic is $7 \times 25=175 \mathrm{mg}$. Since lidocaine is usually available as a $2 \%$ solution, this represents 2 grams in $100 \mathrm{ml}$ of solution or $100 \mathrm{ml}$ of solution will contain 2 grams of local anesthetic (LA).

$1 \mathrm{ml}$ of solution contains $2 / 100=0.02 \mathrm{~g}$ or $20 \mathrm{mg}$ of LA. $1.8 \mathrm{ml}$ of solution ( 1 cartridge) contains $20 \times 1.8=36 \mathrm{mg}$ of LA. If a total of $175 \mathrm{mg}$ can be safely administered to a child weighing $25 \mathrm{~kg}$, this means that $175 / 36=4.8$ or 5 cartridges of lidocaine with epinephrine (1.8 ml of solution) can be safely administered. The use of local and regional analgesia minimizes the need for opioids and sedatives, which is beneficial for aggressive postoperative physical therapy [20].

Table 1 Maximum Dose -- Lidocaine Without Adrenaline [20]

\begin{tabular}{|l|l|l|}
\hline Patient Weight (kg /lb) & mg & No. of cartidges \\
\hline $10 / 23$ & 44 & 1.2 \\
\hline $15 / 34.5$ & 66 & 1.8 \\
\hline $20 / 46$ & 88 & 2.4 \\
\hline $25 / 57.5$ & 100 & 2.7 \\
\hline $30 / 69$ & 132 & 3.6 \\
\hline $40 / 92$ & 176 & 4.8 \\
\hline $50 / 115$ & 220 & 6.1 \\
\hline $60 / 138$ & 264 & 7.3 \\
\hline $70 / 161$ & 300 & 8.3 \\
\hline
\end{tabular}

\subsection{General anesthesia}

General anesthesia is problematic because the simultaneous administration of anticholinergic drugs further aggravates the dryness of the airways.

When it is necessary for dental treatment, patients must be hospitalized and managed by an anesthesiologist. In addition, the dentist must consult the patient's physician before administering the treatment. General anesthetics for adult patients with lung disease should also be used with caution. Mild chronic obstructive pulmonary disease (COPD) or restrictive lung disease is usually not a problem. Moderate to severe lung disease can be aggravated and escalate into severe respiratory distress when inhalation anesthetics are used. This is particularly true of intravenous drugs that depress the respiratory center of the central nervous system, with anticholinergic drugs that may be administered during intubation [12]. 


\section{Postoperative analgesia}

Chronic pain is a common problem in cystic fibrosis, particularly as the patient population ages [21]. Appropriate analgesia may be a concern to prevent any effects of postoperative pain limiting clearance of respiratory secretions. A number of factors may influence drug pharmacokinetics in CF, including malabsorption, a hypermetabolic state, and significant renal or hepatic dysfunction [22]. To our knowledge, at present, no cystic fibrosis database includes all uses of anti-inflammatory drugs in cystic fibrosis.

Some patients may require higher doses of analgesics to achieve adequate pain relief. In the absence of significant liver disease, paracetamol use is generally well tolerated and not associated with adverse effects. Similarly, nonsteroidal antiinflammatory drugs can be used judiciously, when necessary, but are not used in patients with co-existing renal disease or known broncho-reactivity to these agents.

Long-term corticosteroid therapy is not recommended in cystic fibrosis because of its deleterious side effects: growth retardation, osteoporosis, cataract, diabetes, etc. In addition, corticosteroids may promote Pseudomonas aeruginosa colonization. Therefore, any prescription of oral corticosteroids, even for a short period, must be made under close supervision of the patient, paying particular attention to the risk of decompensation of diabetes [22].

Opioid analgesics are generally less appropriate because of their potential to reduce respiratory drive-in patients with severe disease. A more important factor for all patients, however, is the negative effect of opioids on bowel motility, which puts patients at risk for bowel obstruction. When administered cautiously, opioids have been shown to be effective, so it is imperative to provide appropriate counseling to patients to prevent the potentially serious adverse event[4].

\section{Conclusion}

Preventive dental treatment is always essential for children with cystic fibrosis. Planning invasive dental treatment requires close liaison with the child's medical team. There is a need to develop standards to consider when planning dental treatment for individuals with this genetic disease.

\section{Compliance with ethical standards}

\section{Acknowledgments}

The author would like to thank all the contributors to the realization of this paper.

\section{Disclosure of conflict of interest}

The authors declare no conflicts of interest regarding the publication of this paper.

\section{References}

[1] E O'Keefe. « Are children and adolescents with cystic fibrosis at lower risk of caries?: Question: What is the prevalence of caries in children with cystic fibrosis (CF)? », Evid. Based Dent. juin 2014; 15(2): 46-47.

[2] Da Silva Modesto, Karine Barros, et al. « Salivary flow rate and biochemical composition analysis in stimulated whole saliva of children with cystic fibrosis ». Arch Oral Biol. 2015 Nov;60(11):1650-4.

[3] C Harris, G Smith. « Cystic fibrosis in paediatric dental patients », Dent. Nurs. 2013; 9(8): 464-468.

[4] Harrington N, Barry PJ, Barry SM. « Dental treatment for people with cystic fibrosis ». Eur Arch Paediatr Dent. 2016 Jun;17(3):195-203.

[5] S Peker, S Mete, Y Gokdemir, B Karadag, B Kargul. « Related factors of dental caries and molar incisor hypomineralisation in a group of children with cystic fibrosis », Eur. Arch. Paediatr. Dent. 2014; 15(4): $275-280$.

[6] K Herman, M Kowalczyk-Zając, T Pytrus. « Oral cavity health among cystic fibrosis patients: Literature overview », Adv. Clin. Exp. Med. Oct 2017; 26(7): 1147-1153.

[7] EW Sarvas, CE Huebner, JM Scott, JKM Aps, DL Chi. « Dental utilization for Medicaid-enrolled children with cystic fibrosis: DENTAL UTILIZATION FOR CHILDREN WITH CF », Spec. Care Dentist. 2016; 36(6): 315-320. 
[8] T Pawlaczyk-Kamieńska, R Śniatała, H Batura-Gabryel, M Borysewicz-Lewicka, S Cofta. « Periodontal Status and Subgingival Biofilms in Cystic Fibrosis Adults », Pol. J. Microbiol. 2019; 68(3): 377-382.

[9] Genevois A, et al. « Bacterial colonization status of cystic fibrosis children's toothbrushes: A pilot study ». Arch Pediatr. Dec 2015; 22(12): 1240-6.

[10] R Abu-Zahra, N Antos, T Kump, M Angelopoulou. « Oral health of cystic fibrosis patients at a north american center: A pilot study », Med. Oral Patol. Oral Cirugia Bucal. 2019; e379-e384

[11] M Atar, EJ Körperich. « Systemic disorders and their influence on the development of dental hard tissues: A literature review », J. Dent. 2010; 38(4): 296-306.

[12] Rivas Caldas, Rocio, et al. « Pseudomonas aeruginosa and Periodontal Pathogens in the Oral Cavity and Lungs of Cystic Fibrosis Patients: a Case-Control Study ». J Clin Microbiol. 2015 Jun; 53(6): 1898-907.

[13] JA Weddell, BJ Sanders, JE Jones. « Dental Problems of Children with Special Health Care Needs », in McDonald and Avery's Dentistry for the Child and Adolescent, Elsevier. 2016; 513-539.

[14] KELLY, Joseph P. et SHELLER, Barbara L. « A Systematic Approach to Creating a Dental Home for Children with Medical Complexities. In: Dental Care for Children with Special Needs». Springer, Cham, 2019. p. 27-50.

[15] T Pawlaczyk-Kamieńska, M Borysewicz-Lewicka, R Śniatała, H Batura-Gabryel, S Cofta. « Dental and periodontal manifestations in patients with cystic fibrosis - A systematic review », J. Cyst. Fibros., p. S1569199318309354. nov. 2018.

[16] Sanjay H. Chotirmall, Catherine M. Greene, Noel G. McElvaney, «Candida species in cystic fibrosis: A road less travelled ». Medical Mycology, Volume 48, Issue Supplement_1, November 2010,

[17] Bush A, Bilton D, Hodson M. (Eds.). « Hodson and Geddes' Cystic Fibrosis ». CRC Press. 2015.

[18] DA FONSECA, Marcio A. « Oral and dental care of local and systemic diseases ». Pediatr. Dent. 2019; 66-76. e2.

[19] EW Sarvas, CE Huebner, JM Scott, JKM Aps, DL Chi. « Dental utilization for Medicaid-enrolled children with cystic fibrosis: DENTAL UTILIZATION FOR CHILDREN WITH CF », Spec. Care Dentist. 2016; 36(6): 315-320.

[20] DECLOUX, Derek et OUANOUNOU, Aviv. « Local anaesthesia in dentistry: a review ». Int. Dent. J, 2021.

[21] RAVILLY, Sophie, LE ROUX, Evelyne, BELLIS, Gil, et al. « Epidémiologie et physiopathologie de la mucoviscidose ». Revue francophone des laboratoires, 2007, vol. 2007, no 397, p. 25-36.

[22] JARRY-LACOMBE, Hélène. "Les traitements de la mucoviscidose : de la prise en charge symptomatique aux nouvelles thérapeutiques ». 2016. THÈSE POUR LE DIPLÔME D'ÉTAT DE DOCTEUR EN PHARMACIE. 